\title{
A Neural Signature of Affiliative Emotion in the Human Septohypothalamic Area
}

\author{
Jorge Moll, ${ }^{1}$ Patricia Bado, ${ }^{1}$ Ricardo de Oliveira-Souza, ${ }^{1,2}$ Ivanei E. Bramati, ${ }^{1}$ Debora 0. Lima, ${ }^{1}$ Fernando F. Paiva, \\ João R. Sato, ${ }^{1,3}$ Fernanda Tovar-Moll, ${ }^{1,4}$ and Roland Zahn ${ }^{1,5}$ \\ ${ }^{1}$ Cognitive and Behavioral Neuroscience Unit, D’Or Institute for Research and Education (IDOR), 22281-100, Rio de Janeiro, Brazil, ${ }^{2}$ Gaffréee Guinle \\ University Hospital, Federal University of the State of Rio de Janeiro, 20520-054, Rio de Janeiro, Brazil, ${ }^{3}$ Center for Mathematics, Computation, and \\ Cognition, Universidade Federal do ABC, 09020-040, Santo André, Brazil, ${ }^{4}$ Instituto de Ciências Biomédicas, Universidade Federal do Rio de Janeiro, 21941 - \\ 902, Rio de Janeiro, Brazil, and 5University of Manchester and Manchester Academic Health Sciences Centre, School of Psychological Sciences, Oxford \\ Road, M13 9PL, Manchester, United Kingdom
}

Comparative studies have established that a number of structures within the rostromedial basal forebrain are critical for affiliative behaviors and social attachment. Lesion and neuroimaging studies concur with the importance of these regions for attachment and the experience of affiliation in humans as well. Yet it remains obscure whether the neural bases of affiliative experiences can be differentiated from the emotional valence with which they are inextricably associated at the experiential level. Here we show, using functional MRI, that kinship-related social scenarios evocative of affiliative emotion induce septal-preoptic-anterior hypothalamic activity that cannot be explained by positive or negative emotional valence alone. Our findings suggest that a phylogenetically conserved ensemble of basal forebrain structures, especially the septohypothalamic area, may play a key role in enabling human affiliative emotion. Our finding of a neural signature of human affiliative experience bears direct implications for the neurobiological mechanisms underpinning impaired affiliative experiences and behaviors in neuropsychiatric conditions.

\section{Introduction}

The capacity to develop and sustain social bonds ("social attachment") is an ubiquitous characteristic of mammals that promotes group cohesion and cooperation (Insel and Young, 2001; Fehr and Fischbacher, 2004). Research in rodents shows that attachment-dependent behaviors, such as pair-bonding and maternal nurturing (Insel and Young, 2001; Stack et al., 2002), are organized by subcortical structures known to support generic systems for emotion and motivation (i.e., reward vs punishment, appetitive vs aversive) (Berridge and Robinson, 2003; Insel and Fernald, 2004). The key subcortical structures involved in social attachment - the preoptic-anterior hypothalamus, septal nuclei, amygdala, ventral striatum, and bed nucleus of the stria terminalis (Nelson and Panksepp, 1998; Insel and Young, 2001)—are densely interconnected with primary olfactory areas involved in the regulation of species-specific social behaviors through chem-

\footnotetext{
Received Dec. 29, 2011; revised July 17, 2012; accepted July 19, 2012

Author contributions: J.M. and R.Z. designed research; J.M., P.B., I.E.B., D.0.L., F.F.P., and F.T.-M. performed research; I.E.B. contributed unpublished reagents/analytic tools; J.M., P.B., I.E.B., J.R.S., F.T.-M., and R.Z. analyzed data; J.M., R.d.O.-S., F.T.-M., and R.Z. wrote the paper.

This work was supported by Fundação de Amparo á Pesquisa do Estado do Rio de Janeiro (Pronex and Instituto Nacional de Neurociência Translacional grants), Coordenação de Aperfeiçoamento de Pessoal de Nível Superior, Conselho Nacional de Desenvolvimento Científico e Tecnológico, and by intramural grants from IDOR, Brazil. R.Z. was supported by Stepping Stones and an MRC clinician scientist award (G0902304). We thank Aniela Improta França for her help in developing and balancing the written stimuli according to psycholinguistic variables.

The authors declare no competing financial interests.

Correspondence should be addressed to Jorge Moll, Cognitive and Behavioral Neuroscience Unit, IDOR, Rua Diniz Cordeiro, 30, $3^{\circ}$ andar, 22281-100, Rio de Janeiro, Brazil. E-mail: jorge.moll@idor.org.

DOI:10.1523/JNEUROSCI.6508-11.2012

Copyright $\odot 2012$ the authors $\quad 0270-6474 / 12 / 3212499-07 \$ 15.00 / 0$
}

ical signaling (Nelson and Panksepp, 1998; Eisthen, 2002), as well as with medial prefrontal, anterior cingulate, and temporopolar cortices (Nauta and Haymaker, 1969).

In humans, social attachment is inextricably associated with feelings of warmth or tenderness ("affiliative emotion"), which can be subjectively distinguished from nonaffiliative emotional experiences (Depue and Morrone-Strupinsky, 2005; MorroneStrupinsky and Lane, 2007). To date, however, it remains unclear whether there are neural signatures of affiliative feelings beyond those associated with positive and negative hedonic value (Berridge and Robinson, 2003; Insel and Fernald, 2004; Liu et al., 2011). Supporting this hypothesis, focal lesions of rostromedial basal forebrain structures, including the septohypotalamic area, can produce predatory aggression and changes in sexual behavior in humans and animals (Gorman and Cummings, 1992). Furthermore, stimulation of this region elicits pleasurable experiences and prosocial behavior (Bishop et al., 1963; Andy and Stephan, 1968; Irvin et al., 1990). In addition, functional neuroimaging studies in humans have implicated these subcortical structures in individual differences in attachment styles (Gillath et al., 2005), mother-infant bonding (Strathearn et al., 2009), maternal (Bartels and Zeki, 2004) and romantic (Aron et al., 2005) love, and in altruism, cooperation, and trust (Moll et al., 2006; Krueger et al., 2007; Koscik and Tranel, 2011). Thus, converging evidence suggests that a phylogenetically conserved ensemble of basal forebrain structures plays a central role in the organization of social attachment across species (Nelson and Panksepp, 1998).

Despite these lines of evidence, so far no study has attempted to experimentally disentangle affiliative experience from general 
emotional valence. To address this hypothesis, we compared brain responses to affiliative and nonaffiliative social scenarios associated with either positive or negative emotional valence. We hypothesized that a basal forebrain region encompassing the septal/preoptic-anterior hypothalamic structures (Septal/aHyp) would be engaged by the affiliative content of the scenarios, above and beyond general emotional valence. We also hypothesized that, given the purported role of the subgenual cortex and the frontopolar cortex (FPC) in prosocial emotions (Moll et al., 2006, 2011; Krueger et al., 2007; Zahn et al., 2009), these structures would also be recruited by affiliative stimuli.

\section{Materials and Methods}

Thirty adult healthy volunteers ( 16 women; age $=27.3 \pm 9$ years; education $=15.8 \pm 2.6$ years) participated in a pilot study designed to validate the stimulus battery according to the experimental variables of interest. The resulting stimulus set was used in an independent fMRI study with 34 subjects who had not participated in the pilot study. Data from seven participants were discarded before analysis due to signal dropouts in the basal forebrain. The final dataset included 27 righthanded participants. Participants were recruited from local university and institutional settings through word-of-mouth or e-mail lists,comprising university students and staff personnel. Participants had a mean of $1.7 \pm 1.2$ siblings and $0.3 \pm 0.8$ children (i.e., most had siblings, and only 5 of the 27 had children). Men and women did not significantly differ in number of siblings $(p>0.51)$ or children $(p>0.55)$ they had at the time of the study. There were no statistical differences between men's and women's ages ( $28.8 \pm 5.8$ vs $27.1 \pm 4.9$ years, respectively; $p>0.37$ ) or years of education $(16.7 \pm 2.3$ vs $17.1 \pm 2.1$ years, respectively; $p>$ 0.68). All participants were native Brazilian-Portuguese speakers with normal or corrected-to-normal vision, no history of psychiatric or neurological disorders, and none of them were taking centrally active medications. Written informed consent was obtained from all participants, and the study was approved by the D'Or Institute Ethics and Scientific Committee (Study \#128/08).

Stimulus and task design. The initial stimulus set comprised 280 social scenarios consisting of two short syntactically equivalent sentences with 15-18 words connected with an "and" or "but," which were presented one at a time to 30 adult volunteers on a computer screen. Participants used the mouse to rate the amount of positive or negative valence associated with each stimulus on a 15-point Likert scale ranging from "very unpleasant" ( -7$)$ to "neutral" $(0)$ to "very pleasant $(+7)$," and how much "care/tenderness" they felt for the recipient of the action in a given social scenario on a 10-point Likert scale ranging from "absent" to "very intense." Piloting evidence obtained in our lab indicated that the Portuguese words "ternura" (tenderness) and "carinho" (care) best captured the affiliative emotional experiences of attachment. These expressions possess a similar meaning of the English expressions "warm feelings" or "tender feelings," which have been validated as good descriptors of affiliative feelings (Morrone-Strupinsky and Lane, 2007). As in English, these Portuguese expressions can be used both in emotionally positive or negative settings (e.g., tender feelings toward a loved one in a context of joy or sorrow), and thus are not restrictive in terms of valence (as, for example, "compassion," which implies warm feelings toward a suffering individual). For these reasons, affiliative emotion was gauged by the degree to which each participant reported feelings of care/tenderness toward the "object of attachment" of each scenario. For example, in the scenario: "You read a storybook to your young child and he/she quietly fell asleep on your lap," the object of attachment is "your young child." We selected a subset of 200 stimuli ( 80 affiliative, 80 nonaffiliative, 40 neutral) based on ratings on the above dimensions and on proper balancing across all experimental conditions (affiliativepositive, affiliative-negative, nonaffiliative-positive, nonaffiliativenegative and neutral), so that (1) the affiliative condition had high care/tenderness levels, (2) the affiliative and nonaffiliative conditions had equivalent levels of positively and negatively valenced stimuli, and (3) the neutral condition was low in care/tenderness and neutral emotional valence (for a sample of stimuli, see Table 1). To reduce the pos-
Table 1. Samples of stimuli belonging to the affiliative (positive and negative valence) and nonaffiliative (positive and negative valence) subcategories, as well as to the emotionally neutral category

Experimental subconditions Sample stimuli

Affiliative-positive

You taught your son to ride the bike and he came to thank you with a hug.

You and your mom sat on the porch and talked for a long time about old times.

You learned that your mother won a cooking contest and was very happy.

You put together your old pictures from childhood and made an album for your mother.

Nonaffiliative-positive You delivered a beautiful speech and the audience stood up to applaud you.

You went to a rock concert of your favorite band and they invited you to the stage.

You were interested in someone and she/he made some nice remarks about you.

You made some remarks in a meeting and your boss was impressed.

Affiliative-negative You were distracted and lost your young child in the park.

You went to a family meeting in which your parents announced their divorce.

You forgot your wedding anniversary and your spouse got very disappointed.

You found out that your dad was broke and had just lost his job. Nonaffiliative-negative You were blamed for a problem that was not your fault and lost your job.

You met acquaintances and realized that you were the only one unemployed.

You went to a meeting at work and your boss publicly criticized your mistakes.

You hired an employee and later she/he sued you for no apparent reason.

Neutral

You took a street bus close to your home and handed your ticket to the driver.

You ordered some items from the drugstore and someone delivered them to you.

You got some batteries in a local store and paid the cashier in cash.

You went to have lunch and headed to a nearby restaurant with your co-workers.

sibility that nonaffiliative stimuli elicited affiliative emotion, we purposely avoided the use of situations involving good or bad outcomes to another person in the scenario, which could either result from one's own or other's actions, or from fate (i.e., context that could induce prosocial feelings of sympathy, compassion, admiration, or altruistic joy).

fMRI stimuli and task. The stimulus set used in the fMRI study comprised written scenarios pertaining to three categories: (1) affiliative, associated with kin-based attachment, describing social situations involving one's mother, father, spouse, or offspring; (2) nonaffiliative, describing scenarios that did not involve either relatives or close friends; and (3) emotionally neutral social scenarios. All scenarios depicted social situations in which participants were described in the second person. Participants were asked to vividly imagine themselves as agents of the actions therein portrayed. To assess the influence of valence, half the scenarios were pleasant (positive valence triggered by pleasant outcomes) and half were unpleasant (negative valence). Therefore, four subconditions comprised the affiliative and nonaffiliative scenarios: affiliative-positive, affiliative-negative, nonaffiliative-positive and nonaffiliative-negative. There were 80 affiliative and 80 nonaffiliative stimuli (half with positive and half with negative valence), and 40 neutral stimuli. An LCD display mounted in the scanner room, which was seen by the participant by way of a mirror system adapted to the head coil, was used for stimulus delivery. Stimuli were presented in a pseudo- 


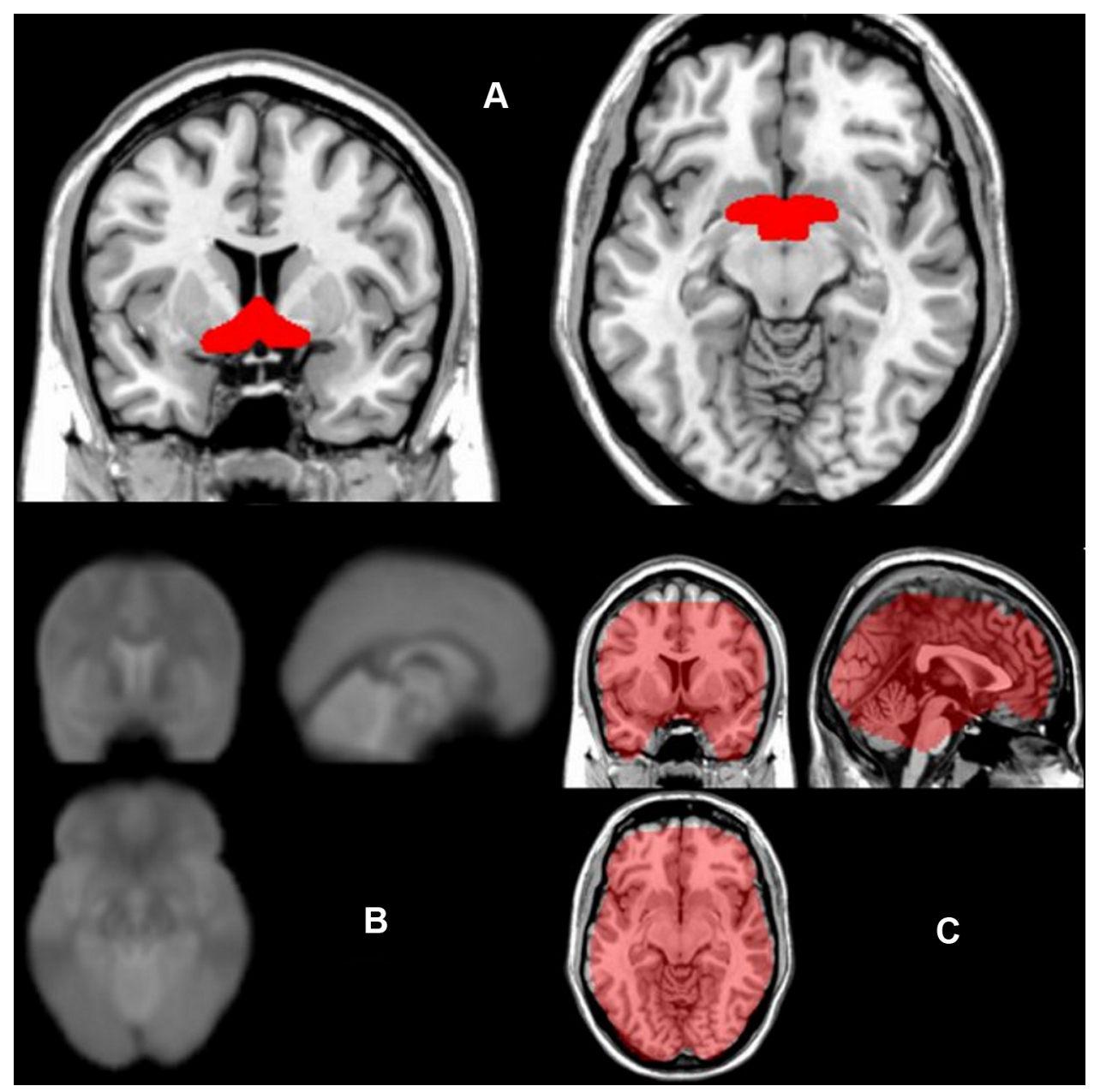

Figure 1. A, Anatomical coverage of the a priori ROI of the septal/preoptic-anterior hypothalamic region, used for small-volume correction for multiple comparisons. $\boldsymbol{B}$, Average echoplanar images from all subjects show preserved signal at the basal forebrain (i.e., no signal dropouts at the individual and group level, except for a small portion of the ventromedial posterior orbitofrontal cortex). C, Binary mask used by SPM8 in the second-level analysis, overlaid on a T1 anatomical template, shows preserved fMRI signal in basal forebrain and prefrontal regions.

randomized order using a slow event-related design. Before each run, a $10 \mathrm{~s}$ countdown was shown. Participants were then presented with a written scenario for $6 \mathrm{~s}$ followed by a screen depicting the words "pleasant" and "unpleasant" side by side and told to make a choice within 3 s by pressing either the right or the left button on an MRI-compatible response pad device (Lumina; Cedrus). Position of the words (right or left) was counterbalanced. This low-demanding task kept participants engaged while avoiding unwanted executive load. A cross-fixation screen followed for an average of $2.25 \mathrm{~s}$ (varying from 1.5 to $3 \mathrm{~s}$, in $250 \mathrm{~ms}$ steps), introducing a temporal jitter to increase the sampling efficiency of the hemodynamic responses (total trial time $=11.25 \mathrm{~s}$ ). We did not use a low-level baseline condition because the mental imagery associated with the typical default state often includes self- and socially-relevant contents. Such activity would overlap that of our target condition in a poorly controlled fashion, thus failing to provide a proper baseline. Before scanning, the task was thoroughly explained to participants and a brief training with five extra scenarios was administered. After scanning, participants rated each stimulus on a computer according to (1) degree of affiliative emotion (care/tenderness) they experienced toward the recipient of the outcomes of the described situations [10-point Likert scales ranging from "absent" (0) to "very intense" (9)], (2) emotional valence [15-point scales ranging from "very unpleasant" $(-7)$ to "very pleasant" (7), with "neutral" on the center], and (3) number of consequences foreseen by the participant to each scenario [10-point scales ranging from "none" (0) to "several" (9) possible consequences] in response to the question "How many possible consequences can you imagine for this scenario?" Ratings of autobiographical scene likeness of stimuli belong- ing to the affiliative, nonaffiliative, and neutral conditions [10-point scales ranging from "I have never experienced a similar situation in my life" (0) to "I have experienced an extremely similar situation in my life" (9)] were also obtained from participants.

Statistical analyses of behavioral data. Results are expressed as means and standard deviations. The behavioral effects of affiliation, valence, and response times were assessed with linear mixed effects models (with subjects entered as a random effect in a general linear model) and $t$ tests for pairwise comparisons. A significance threshold $(\alpha)$ of 0.05 , twotailed, was adopted for all statistical tests.

Image acquisition and analysis. Functional images were acquired with a 3T Achieva scanner (Philips Medical Systems) using a T2*-weighted echoplanar (BOLD contrast) sequence $(\mathrm{TR}=2000 \mathrm{~ms}$, $\mathrm{TE}=22 \mathrm{~ms}$, matrix $=96 \times 96, \mathrm{FOV}=240 \mathrm{~mm}$, flip angle $=90^{\circ}$, slice thickness $=3$ $\mathrm{mm}$, 39 slices; 287 volumes per run, 4 runs). Total functional scanning time was $\sim 41 \mathrm{~min}$. Before each run, five dummy volumes were collected for T1 equilibration purposes. A SENSE factor of 1.5 and dynamic stabilization were additionally used. These parameters were based on careful sequence optimization to maximize temporal signal-to-noise (Bodurka et al., 2007) in brain regions that normally suffer from magnetic susceptibility effects, including the basal forebrain areas and ventromedial regions of the prefrontal cortex. To ensure coverage of the basal forebrain regions that were expected to be involved in affiliative emotion, the echoplanar imaging (EPI) datasets from all participants were inspected. Figure 1 shows the mean EPI from all participants, as well as the coverage of the SPM8 implicit mask, which only includes brain regions that had 
adequate signal in the source EPI images of every single participant. High-resolution anatomical images were acquired with a 3D turbo fieldecho $\mathrm{T} 1$-weighted sequence $(\mathrm{TR}=13 \mathrm{~s}, \mathrm{TE}=1.4 \mathrm{~s}$, matrix $=256 \times 356$, $\mathrm{FOV}=240 \mathrm{~mm}$, slice thickness $=1 \mathrm{~mm}, 140$ slices). Head motion was restricted by using foam padding and straps over the forehead and under the chin (estimated translation and rotation parameters were inspected and never exceeded $2 \mathrm{~mm}$ or $2^{\circ}$ ). Statistical Parametric Mapping (SPM; www.fil.ion.ucl.ac.uk/spm/software/spm8) and the general linear model were used for image analysis (Friston et al., 1995; Worsley and Friston, 1995). Additional analyses entering affiliation and valence ratings as parametric modulators in the design matrix were not performed because some participants showed ceiling- or floor-level ratings with too little variability on either affiliation or valence in some runs (preventing SPM from modeling parametric effects). Therefore, categorical modeling of valence and affiliation was most appropriate. Categorical contrasts were created by condition-specific events with a canonical hemodynamic response function. All functional datasets underwent registration and 12parameter affine normalization, and transformed into the standard MNI space using a $2 \times 2 \times 2$ isotropic resolution. Functional data were smoothed using an $8 \mathrm{~mm}$ FWHM Gaussian spatial kernel. High-pass filtering (128s) and cubic detrending were used to remove unwanted low frequencies from the fMRI time series (Macey et al., 2004). For the within-subject, first-level analysis, the entire trial duration for each condition-specific event was modeled ( $6 \mathrm{~s}$ of scenario presentation plus $3 \mathrm{~s}$ window for subject response) and convolved with a canonical hemodynamic response function. Statistical effects were calculated on the second level by using a random-effects model. Significance was determined by using either a voxel-level FWE-corrected $p<0.05$ over the whole brain or over a priori predicted regions of interest (ROIs). A priori ROIs of the Septal/aHyp region and of the anterior prefrontal cortex (encompassing the FPC) were derived from an independent study (Zahn et al., $2009 \mathrm{~b}$ ) and modified by extending the hypothalamic ROI to the septal area of the basal forebrain. As for the subgenual cortex ROI, an a priori coordinate $(0,26,-5)$ was derived from averaging center coordinates from local maxima from three independent studies (Moll et al., 2006; Zahn et al., 2009a,b). The search volume consisted of a $10 \mathrm{~mm}$ sphere centered on this coordinate. Post hoc analyses of beta values extracted from local maxima were conducted using a mixed-effects ANOVA (general linear model; affiliation and valence modeled as fixed effects, and subjects as a random effect). All reported coordinates are in Montreal Neurological Institute space. Labeling of brain regions was based on standard anatomical criteria (Mai and Paxinos, 2004).

\section{Results}

\section{Behavioral results}

As planned, affiliation ratings were significantly higher for the affiliative than for the nonaffiliative condition ( $p<0.001$; Fig. 2, top). The average valence of all conditions pooled together was close to zero $(0.10 \pm 5.78)$, indicating that positive and negative conditions were equivalent in emotional arousal (Fig. 2, bottom). Valence ratings were highly congruent $(>95 \%)$ with the intended category within each of the four main subconditions (affiliative-positive, affiliative-negative, nonaffiliative-positive, nonaffiliative-negative), as well as the neutral condition. Response times did not statistically differ among the four main subconditions $(p>0.18)$. There were no differences between men and women in their overall ratings of affiliation $(p>0.76)$ and valence $(p>0.16)$ of the affiliative and nonaffiliative conditions, which also did not differ in terms of imagined number of possible outcomes $(p=0.12)$.

\section{Functional MRI results}

A direct comparison between the affiliative and the nonaffiliative conditions revealed robust activations in (1) a neural continuum that encompassed the preoptic area, ventromedial hypothalamus, and medial septum (mainly the vertical limb of the diagonal band of Broca), i.e., the Septal/aHyp region; and (2) in the medial FPC (BA 10; Fig. 3, Table 2). A second contrast to directly com-
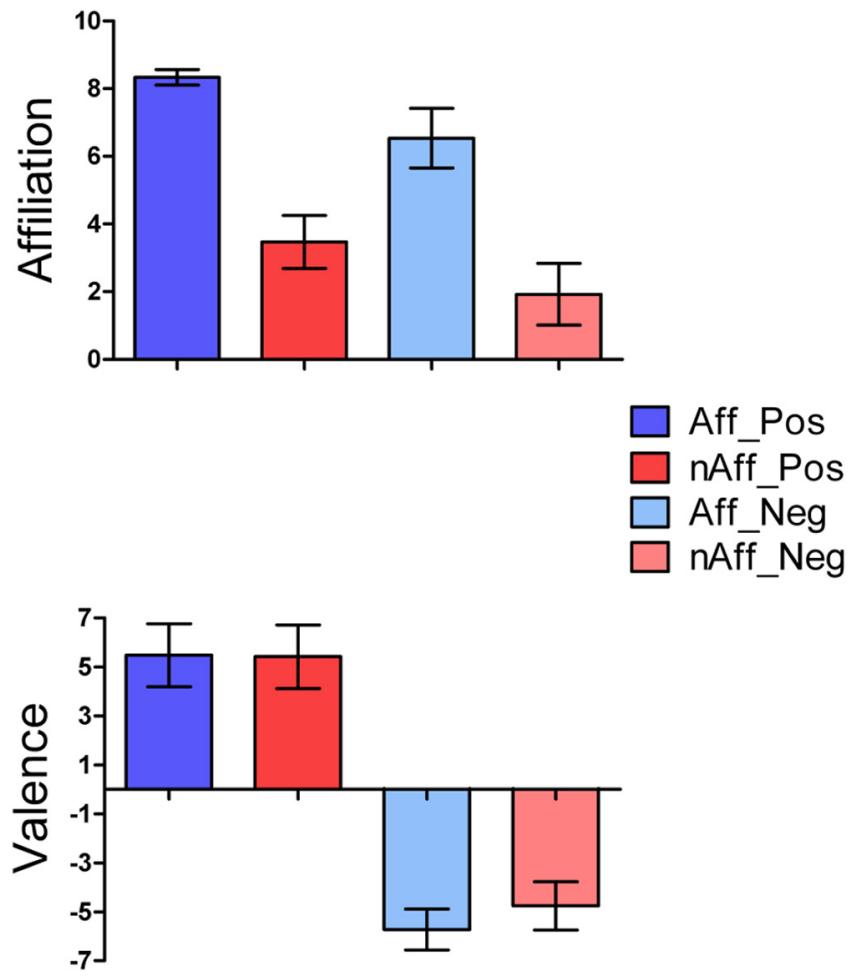

Figure 2. Top, Affiliative emotion ratings (care/tenderness) for the affiliative and nonaffiliative main experimental conditions, broken into the four subconditions (Aff_Pos, affiliative-positive; nAff_Pos, nonaffiliative-positive; Aff_Neg, affiliative-negative; nAff_Neg, nonaffiliative-negative). The affiliative condition showed significantly higher care/tenderness ratings than the nonaffiliative condition ( $n=80 /$ group; $t=57.57, p<$ $0.0001)$. Bottom, Comparison of positive and negative emotional valence ratings between affiliative and nonaffiliative main conditions, broken into the four emotional subconditions. Pairwise comparisons between affiliative versus nonaffiliative main conditions (affiliative-positive vs nonaffiliative-positive and affiliative-negative vs nonaffiliativenegative) did not reveal statistically significant differences ( $n=40 /$ group; all $p>0.05$, mixed-effects ANOVA, entering subjects as a random variable). Error bars represent SDs.

pare the affiliative and neutral conditions revealed that the differential activity observed in the affiliative condition resulted from more activation in these brain regions, rather than less deactivation compared with the neutral condition (FWE-corrected, $p<$ 0.05 over the whole brain). A confirmatory analysis performed on the beta values extracted from Septal/aHyp and FPC local maxima for the four subconditions (affiliative-positive, affiliativenegative, nonaffiliative-positive, nonaffiliative-negative) rejected the hypothesis that affiliation and valence interacted in the Septal/aHyp $(p>0.45)$ and in the FPC $(p>0.84)$. This is in agreement with the lack of significant interactions produced by the SPM8 factorial design at the whole-brain level using uncorrected thresholds $(p<0.005)$. This analysis also revealed that, in addition to the main effect of affiliation in the Septal/aHyp ( $p=$ $0.017)$ and in the FPC $(p>0.001)$, there was a main effect of valence. Interestingly, whereas the Septal/aHyp area showed increased responses to positive emotional valence ( $p=0.011)$, the FPC showed higher activation to negative emotional valence $(p<0.001)$. The subgenual cortex exhibited no activation even at lenient thresholds. Nonpredicted effects for the affiliative condition were observed in the precuneus (BA 7/23) and in the left posterior superior temporal sulcus (STS; BA 21/22; Fig. 3). Further control analyses demonstrated that these effects were not dependent on participants having children of their own or on the level of autobiographical resemblance of the hypothetical scenarios used in the affiliative and nonaffiliative conditions. 


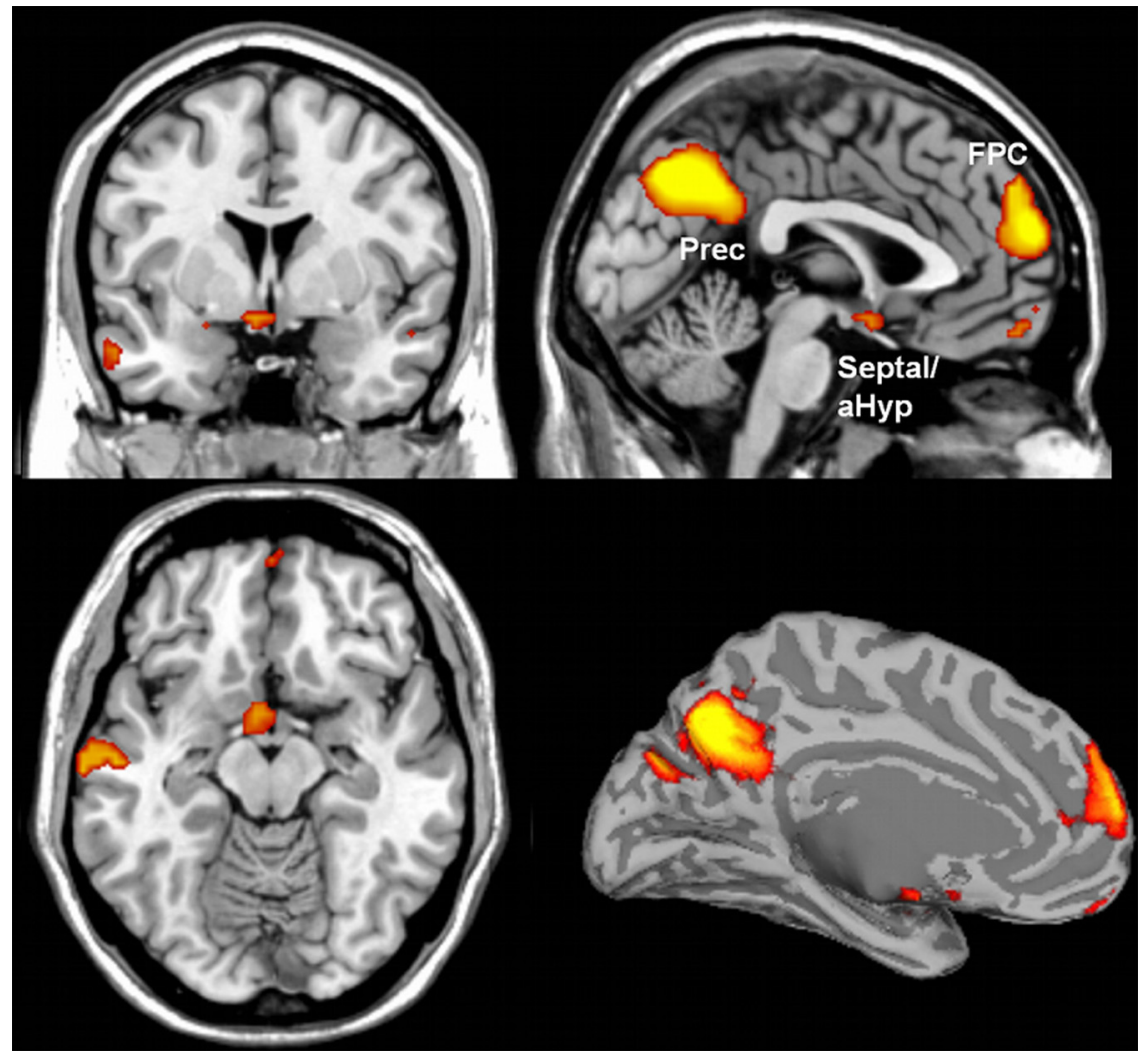

Figure 3. Activation of the septal/preoptic-anterior hypothalamic and medial FPC, predicted a priori, as well as in the left posterior superior temporal sulcus region (data not shown) and precuneus (Prec), observed in the affiliative versus nonaffiliative contrast. To provide a better visualization of the spatial extent of the significantly activated clusters, statistical maps are displayed at uncorrected $p=0.005$, minimum cluster size $=5$ voxels. These clusters survived FWE correction for multiple comparisons $(p<0.05)$ either at the whole-brain level or over an a priori anatomical region of interest (Table 2; additional clusters showing in this map did not survive FWE correction). Activation results overlaid on a segmented and inflated brain template are shown at the right inferior quadrant.

Table 2. Brain activation in the affiliative versus nonaffiliative contrast

\begin{tabular}{|c|c|c|c|c|c|c|}
\hline & \multirow{2}{*}{$\begin{array}{l}\text { Cluster } \\
\text { size }\end{array}$} & \multirow{2}{*}{$\begin{array}{l}z \\
\text { value }\end{array}$} & \multirow{2}{*}{$\begin{array}{l}p \\
\text { (uncorrected) }\end{array}$} & \multicolumn{3}{|c|}{ MNI local maxima } \\
\hline & & & & $x$ & $y$ & $Z$ \\
\hline Medial frontopolar cortex (BA 10)* & 839 & 4.98 & $<0.001$ & 0 & 62 & 19 \\
\hline Precuneus (BA 7/23)* & 661 & 6.21 & $<0.001$ & -3 & -64 & 37 \\
\hline Posterior superior temporal sulcus (BA 21/22)* & 385 & 5.59 & $<0.001$ & -54 & -70 & 22 \\
\hline Septal/anterior hypothalamic region ${ }^{\dagger}$ & 046 & 3.40 & $<0.001$ & -3 & 2 & -14 \\
\hline
\end{tabular}

All reported clusters survived either FWE correction for multiple comparisons $(p<0.05)$ over the whole brain $\left(^{*}\right)$ or over an a priori anatomical ROI $\left(^{\dagger}\right)$, using a minimum cluster size of five voxels.

Analyses of the effects of positive and negative emotional valence [(affiliative-positive + nonaffiliative-positive) vs (affiliativenegative + nonaffiliative-negative)] were also conducted. Positive valence, compared with negative valence, was associated with bilateral responses in the medial sectors of the ventral striatum and medial orbitofrontal cortex (whole brain, FWE-corrected, $p<0.05$ ). Negative valence evoked activation bilaterally in the dorsomedial prefrontal cortex (BA 8/9), lateral orbitofrontal cortex/inferior frontal gyri, and neighboring anterior insula (BA 45/47/48; whole brain, FWE-corrected, $p<0.05$; Fig. 4 ). There were no significant interaction effects between affiliation and valence in any of these regions at the whole-brain level using uncorrected thresholds $(p<0.005)$.

\section{Discussion}

The present investigation shows that the septal/preoptic-anterior hypothalamic area (Septal/aHyp) is engaged by affiliative stimuli.
These results could not be attributed to the elicitation of either positive or negative emotion alone, thus indicating that these structures may play a central role for affiliative states beyond the effect of hedonic value. Our findings reveal that the same Septal/aHyp structures that are involved in the neural organization of affiliative behaviors across several species are also engaged by affiliative experiences in humans (Nelson and Panksepp, 1998; Goodson and Wang, 2006).

Supporting our findings, in a study of first-time new mothers, Strathearn et al. (2009) observed greater hypothalamic activation in the brain of mothers who developed secure attachment with their infants, whereas insecure/dismissing mothers showed greater activation of the insula. This study, however, was not designed to control for emotional valence and therefore it is not possible to rule out that the higher hypothalamic activation observed in the securely attached mothers was solely driven by emotional valence.

The observation that affiliative experience can be partially dissociable from general hedonic experience (Depue and Morrone-Strupinsky, 2005; MorroneStrupinsky and Lane, 2007) is in agreement with our behavioral findings in that kinship-related affiliative scenarios evoke higher levels of tender feelings than nonaffiliative ones with equivalent levels of positive or negative emotional valence. We also found that ventral striatum and medial orbitofrontal cortex activity was modulated by positive emotional valence, whereas the lateral orbitofrontal cortex and adjacent anterior insula responded more strongly to negative emotion, findings that are in agreement with a large body of evidence on the role of these regions in coding for emotional arousal in humans (for review, see Liu et al., 2011). The fact that brain regions classically implicated in positive or negative emotional experiences were not more engaged by affiliative than by nonaffiliative stimuli further supports the special role of the Septal/aHyp in affiliative experience. Interestingly, Septal/aHyp responses to affiliative stimuli were higher in the context of positive valence. Although further studies are needed, we speculate that this effect indicates a more specific role of this region in positive social reinforcement. This is in agreement with imaging studies reporting activity in this brain region in tasks involving interpersonal trust and charitable donations (Moll et al., 2006; Krueger et al., 2007; Hsu et al., 2008).

We also found that the FPC was more active in the affiliative than in the nonaffiliative condition. Although the exact role of the FPC in social affiliation is still poorly understood, previous functional MRI and clinical studies have specifically implicated this region in enabling prosocial moral feelings (Moll et al., 2007, 2011; Krajbich et al., 2009) and decisions (Moll et al., 2006; Krueger et al., 2007). The anterior hypothalamic and neighboring rostrobasal forebrain structures that were more activated in the affiliative conditions of the present study stand among the few 
that sustain reciprocal direct connections with the FPC in the primate brain, including in humans (Rempel-Clower and Barbas, 1998).

The lack of subgenual cortex response to affiliative stimuli highlights the puzzling role of this region in social cognition and emotion, both in normal individuals and in patients with major depressive disorder (Mayberg et al., 2005; Decety, 2011). Also, the unanticipated activation of the precuneus and STS indicate that these regions are somehow more critically involved in imagined social contexts when affiliative emotions are at play. Further studies are needed to settle these issues.

Current functional MRI techniques are limited in accurately discerning brain activation arising from closely located, small subcortical nuclei. Thus, the brain responses herein described in the Septal/ aHyp area cannot be safely ascribed to specific basal forebrain nuclei (e.g., hypothalamic nuclei, bed nucleus of the stria terminalis, septal nuclei), so it is possible that any of these structures may have contributed to the observed effects. Nonetheless, our reported activation in the Septal/ aHyp area can be safely distinguished from other neighboring regions, such as the medial and cortical amygdaloid nuclei and ventral striatum. Future studies using more refined MRI methods are needed to further define the contribution of subregions of the basal forebrain to affiliative emotion. This will be especially relevant for assessing whether partially distinct subcortical responses are associated with subtypes of social affiliation. Whereas the present study used kinship stimuli in a nondistinctive manner, further studies may explore differences in brain responses evoked by attachment to romantic partners, parents, siblings, offspring, and friends.

The finding of a neural signature for affiliative experience toward kin, independent of hedonic value, holds promise for a better understanding of the mechanisms underlying antisocial or asocial behaviors that comprise the core symptoms of common neuropsychiatric illnesses, such as autism, psychopathy, schizophrenia, and drug addiction. Recognizing the role of a neural circuitry for affiliative experiences beyond the classical brain networks supporting general reward and punishment mechanisms opens new possibilities for diagnosis and treatment of these disorders.

\section{Notes}

Supplemental material for this article is available at http://idor.org/ resources/Moll_et_al_Affiliative_Emotion_JN2012. This material has not been peer reviewed.

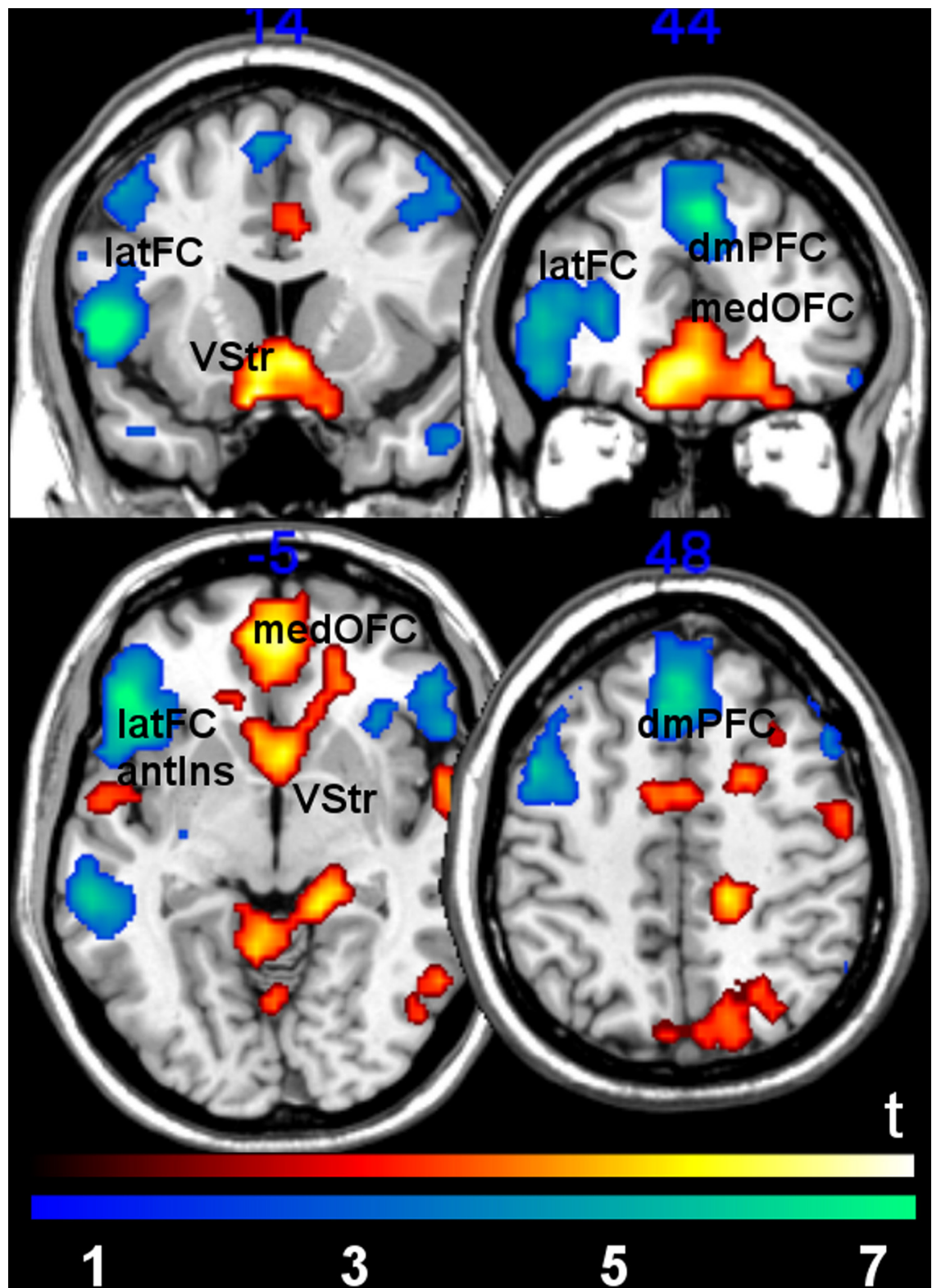

Figure 4. Brain regions associated with positive versus negative conditions (red-yellow) and negative versus positive contrasts (blue-green). Activation of the ventral striatum (VStr) and medial orbitofrontal cortex (med0FC; BA11/32) was observed in the positive versus negative contrast. For the negative versus positive contrast, activation of dorsomedial prefrontal cortex (dmPFC; BA 8/9) and lateral frontal cortex, including the lateral orbitofrontal cortex and inferior frontal gyrus (latFC), as well as the adjoining anterior insula (antlns) was observed (BA 45/47/48). Only regions that survived whole-brain FWE-correction (voxel-level, $p=0.05$ ) are reported. Statistical images displayed at uncorrected threshold $p=0.005$, minimum cluster size $=5$ voxels for visualization purposes.

\section{References}

Andy OJ, Stephan H (1968) The septum in the human brain. J Comp Neurol 133:383-410.

Aron A, Fisher H, Mashek DJ, Strong G, Li H, Brown LL (2005) Reward, motivation, and emotion systems associated with early-stage intense romantic love. J Neurophysiol 94:327-337.

Bartels A, Zeki S (2004) The neural correlates of maternal and romantic love. Neuroimage 21:1155-1166.

Berridge KC, Robinson TE (2003) Parsing reward. Trends Neurosci 26:507-513.

Bishop MP, Elder ST, Heath RG (1963) Intracranial self-stimulation in man. Science 140:394-396.

Bodurka J, Ye F, Petridou N, Murphy K, Bandettini PA (2007) Mapping the MRI voxel volume in which thermal noise matches physiological noise: implications for fMRI. Neuroimage 34:542-549. 
Decety J (2011) The neuroevolution of empathy. Ann N Y Acad Sci 1231:35-45.

Depue RA, Morrone-Strupinsky JV (2005) A neurobehavioral model of affiliative bonding: implications for conceptualizing a human trait of affiliation. Behav Brain Sci 28:313-350.

Eisthen HL (2002) Why are olfactory systems of different animals so similar? Brain Behav Evol 59:273-293.

Fehr E, Fischbacher U (2004) Social norms and human cooperation. Trends Cogn Sci 8:185-190.

Friston KJ, Frith CD, Turner R, Frackowiak RS (1995) Characterizing evoked hemodynamics with fMRI. Neuroimage 2:157-165.

Gillath O, Bunge SA, Shaver PR, Wendelken C, Mikulincer M (2005) Attachment-style differences in the ability to suppress negative thoughts: exploring the neural correlates. Neuroimage 28:835-847.

Goodson JL, Wang Y (2006) Valence-sensitive neurons exhibit divergent functional profiles in gregarious and asocial species. Proc Natl Acad Sci U S A 103:17013-17017.

Gorman DG, Cummings JL (1992) Hypersexuality following septal injury. Arch Neurol 49:308-310.

Hsu M, Anen C, Quartz SR (2008) The right and the good: distributive justice and neural encoding of equity and efficiency. Science 320: 1092-1095.

Insel TR, Fernald RD (2004) How the brain processes social information: searching for the social brain. Annu Rev Neurosci 27:697-722.

Insel TR, Young LJ (2001) The neurobiology of attachment. Nat Rev Neurosci 2:129-136.

Irvin RW, Szot P, Dorsa DM, Potegal M, Ferris CF (1990) Vasopressin in the septal area of the golden hamster controls scent marking and grooming. Physiol Behav 48:693-699.

Koscik TR, Tranel D (2011) The human amygdala is necessary for developing and expressing normal interpersonal trust. Neuropsychologia 49:602-611.

Krajbich I, Adolphs R, Tranel D, Denburg NL, Camerer CF (2009) Economic games quantify diminished sense of guilt in patients with damage to the prefrontal cortex. J Neurosci 29:2188-2192.

Krueger F, McCabe K, Moll J, Kriegeskorte N, Zahn R, Strenziok M, Heinecke A, Grafman J (2007) Neural correlates of trust. Proc Natl Acad Sci U S A 104:20084-20089.

Liu X, Hairston J, Schrier M, Fan J (2011) Common and distinct networks underlying reward valence and processing stages: a meta-analysis of functional neuroimaging studies. Neurosci Biobehav Rev 35:1219-1236.

Macey PM, Macey KE, Kumar R, Harper RM (2004) A method for removal of global effects from fMRI time series. Neuroimage 22:360-366.
Mai JK, Paxinos G (2004) Atlas of the human brain, second edition. San Diego: Elsevier.

Mayberg HS, Lozano AM, Voon V, McNeely HE, Seminowicz D, Hamani C, Schwalb JM, Kennedy SH (2005) Deep brain stimulation for treatmentresistant depression. Neuron 45:651-660.

Moll J, Krueger F, Zahn R, Pardini M, de Oliveira-Souza R, Grafman J (2006) Human fronto-mesolimbic networks guide decisions about charitable donation. Proc Natl Acad Sci U S A 103:15623-15628.

Moll J, de Oliveira-Souza R, Garrido GJ, Bramati IE, Caparelli-Daquer EM, Paiva ML, Zahn R, Grafman J (2007) The self as a moral agent: linking the neural bases of social agency and moral sensitivity. Soc Neurosci 2:336-352.

Moll J, Zahn R, de Oliveira-Souza R, Bramati IE, Krueger F, Tura B, Cavanagh AL, Grafman J (2011) Impairment of prosocial sentiments is associated with frontopolar and septal damage in frontotemporal dementia. Neuroimage 54:1735-1742.

Morrone-Strupinsky JV, Lane RD (2007) Parsing positive emotion in relation to agentic and affiliative components of extraversion. Pers Individ Dif 42:1267-1278.

Nauta WJH, Haymaker W (1969) Hypothalamic nuclei and fiber connections. In: The hypothalamus (Haymaker W, Anderson E, Nauta WJ, eds), pp 136-209. Springfield, Ill: Charles C Thomas.

Nelson EE, Panksepp J (1998) Brain substrates of infant-mother attachment: contributions of opioids, oxytocin, and norepinephrine. Neurosci Biobehav Rev 22:437-452.

Rempel-Clower NL, Barbas H (1998) Topographic organization of connections between the hypothalamus and prefrontal cortex in the rhesus monkey. J Comp Neurol 398:393-419.

Stack EC, Balakrishnan R, Numan MJ, Numan M (2002) A functional neuroanatomical investigation of the role of the medial preoptic area in neural circuits regulating maternal behavior. Behav Brain Res 131:17-36.

Strathearn L, Fonagy P, Amico J, Montague PR (2009) Adult attachment predicts maternal brain and oxytocin response to infant cues. Neuropsychopharmacology 34:2655-2666.

Worsley KJ, Friston KJ (1995) Analysis of fMRI time-series revisitedagain. Neuroimage 2:173-181.

Zahn R, de Oliveira-Souza R, Bramati I, Garrido G, Moll J (2009a) Subgenual cingulate activity reflects individual differences in empathic concern. Neurosci Lett 457:107-110.

Zahn R, Moll J, Paiva M, Garrido G, Krueger F, Huey ED, Grafman J (2009b) The neural basis of human social values: evidence from functional MRI. Cereb Cortex 19:276-283. 